\title{
Functional Dynamics: From Biological Complexity to Translation and Impact in Healthcare Systems
}

\author{
Jonathan A. Lal ${ }^{1 *}$, Ralf Sudbrak², Hans Lehrach ${ }^{2}$ and Angela Brand
}

${ }^{1}$ Institute for Public Health Genomics, Department of Genetics and Cell Biology, School for Oncology and Developmental Biology (GROW), Faculty of Health Medicine and Life Sciences, Maastricht University, The Netherlands

${ }^{2}$ Max Planck Institute for Molecular Genetics, Department of Vertebrate Genomics, Berlin, Germany

\begin{abstract}
Biological complexity at a molecular and physiological level is dynamically translucent and requires a systemwide computational approach to possibly elucidate underlying mechanisms for medical and public health applications. Functional dynamics is ideal to study molecular functions given biological functions are dependent on the dynamic nature of networks it operates within. However, environmental factors significantly affect the molecular dynamics in biology, which still needs to be incorporated in study of functions for medical applicability. Through technological innovation medicine is seeing a potential shift in demand for personalized interventions, which has not been fully realized yet. Also the applicability of functional dynamics' utility seems not visible in healthcare systems. This article addresses the above mentioned issues, challenges in translation/implementation using the example of the "virtual patient" developed through the pilot EU flagship project ICT Future of Medicine, and provides possible solutions and insights of new and existing scientific data, infrastructures and frameworks like the Learning-Adapting-Leveling model to make it feasible including policy-wise by incorporating best practice guidelines developed through the Public Health Genomics European Network and tries to touch upon its consequential impact. As a result, we see that real time integration in healthcare requires early-on involvement of all stakeholders as well as taking into account health policy issues, which is addressed by the proposed Learning-Adapting-Leveling model and the best practice guidelines. Furthermore, environmental factors and exposome properties need to be taken into consideration, which the pilot ICT Future of Medicine has been taken into account. We now possibly see a shift from stratified medicine through personalized medicine and possibly towards individualized medicine. This coupling of the pilot project ICT Future of Medicine by integrating the Learning-Adapting-Leveling model to resolve real-time integration issues and considering policy-wise the best practice guidelines has set the stage for it to potentially revolutionize the healthcare system as a whole.
\end{abstract}

Keywords: Computational biology; Functional dynamics; Information and communication technologies; Public health genomics; Personalized medicine; Systems biology; Genomics; Health technology assessment; Health needs assessment; Health impact assessment; Technology transfer

\section{Introduction}

\section{The complexity of biology}

Over the progression of scientific knowledge and discovery especially in the field of genome-based information and technologies (encompassing all-omics, for example genomics, transcriptomics, proteomics, metabolomics, etc. and their interaction with environmental factors), we come short of understanding the complexity of the highly dynamic molecular human biology with all permutations and combinations of interactions and physiology itself; rather we are only able to grasp the complexity of it. What makes the molecule level biology far complex is that not only does function rely on the molecule (genes, proteins, transcription factors, metabolites, etc.) itself, but also relies with the combination of network of interactions (for example gene-gene, gene-protein, protein-protein, 1 protein- 12 proteins, etc.) it is attached. This can constitute feed-back/forward control [1,2], modularity [3], redundancy [4] and multitasking [5]. The function of operation can also be dependent on the structure of the molecule and/ or the structural stability of the network [6] and localization. Certain molecules can act as genetic switches among others. Inhibiting a molecule (say gene) may not inhibit the function itself, but being linked to a stable network and/or complimentary genes can compensate. Also networks can be unaffected to perturbations as complimentary yet unrelated networks can stand in. In addition to that the combination of the functional molecule and the network itself is required for execution of the function as separated, may not work. Furthermore, large well connected networks are least affected to perturbations compared to not so well connected networks, however the prior can be fragile if the most influential nodes are corrupted. This is all being in a highly dynamic, constantly adapting and changing environment and at different levels (molecular, cellular and tissues). A very nice review to this end has been written by Kitano already in 2002 [7]. What adds to the complexity of the network is that previously unconnected diseases or genes seem related and have not yet been medically classified accordingly [8].

\section{Systems approach}

From the above, what can be understood is that although single gene/protein research is still required to gather information about function and processes, a more holistic approach is needed to elucidate the functional complexity of a system, which is the human. This also

\footnotetext{
*Corresponding author: Jonathan A Lal, Institute for Public Health Genomics, Department of Genetics and Cell Biology, School for Oncology and Developmental Biology (GROW), Faculty of Health Medicine and Life Sciences, Maastricht University, Universiteitssingel 50, 6229 ER Maastricht The Netherlands, Tel: +31-43-388-1704; Fax: +31-43-388-1241; E-mail: j.lal@maastrichtuniversity.nl
}

Received March 28, 2013; Accepted April 16, 2013; Published April 19, 2013

Citation: Lal JA, Sudbrak R, Lehrach H, Brand A (2013) Functional Dynamics: From Biological Complexity to Translation and Impact in Healthcare Systems. J Comput Sci Syst Biol 6: 088-092. doi:10.4172/jcsb.1000104

Copyright: (c) 2013 Lal JA, et al. This is an open-access article distributed under the terms of the Creative Commons Attribution License,which permits unrestricted use, distribution, and reproduction in any medium, provided the original author and source are credited. 
implies for drug discovery and targeting as well as therapy. Through -omics based research, we now know that functionality or execution of a process is dependent upon multiple molecules and networks and no one molecule or network can work on its own, therefore can be viewed as a system. Drug targeting now requires consequences to the network (or so called side-affects). The data generated by this information, especially in biological systems impracticably large, consequentially make it dependent on computational power to store, analyze and interpret it unlike what was previously thought to be localized to the wet lab. This has been evident from the very basic of biological computing in BLAST [9] to data analysis [10,11], among others [1214]. This convergence of biology and computation power has been recently discussed [15], stating the fact that the two fields have been alongside each other for quite some time and rely on each other for inspiration.

Systems biology is defined as an integrative, interdisciplinary approach to biological science that is built around the concept of close integration of computational methods, technology development (including -omics) and global measurement and analysis of biological systems [16], and seem to be the future to this holistic or systemwide approach. Functional dynamics within systems biology as a field on its own greatly corresponds to the study of biological systems as the latter being dynamic is a key in its function. The challenge for functional dynamics and systems biology as a whole is to integrate not just biological complexity previously mentioned above but also take into consideration external environmental interactions with the biological system at different levels which can significantly impact (through mutations, single nucleotide polymorphisms, change in network behavior, etc.) the biological system. From epigenomics/ epigenetics, we now know that genome-based information and its' permutations are not the only factor detrimental in health outcomes but environmental (including chemical, surroundings, lifestyle, social and economic) factors influence the interactome (complete repertories of interactions potentially encoded by the genome) [17], of human molecular physiology through the superimposition of the exposome (combined exposures from all sources that reach the internal chemical environment) [18]. With the exposome being in constant contact with the molecular complex system, one cannot appreciate and predict the functionality of the dynamics involved without taking into account the exposome.

\section{Personalized and individualized}

With the completion of the human genome project and the postgenomics era, which started off with transcriptomics, proteomics and metabolomics among others pointed to the direction of a more individualized or personalized healthcare effort. As the user or patient became aware of knowledge through the influx of information present on the internet and (social) media and related online tools and digital libraries, the user demands personalized or preferentially individualized approaches towards his/her medical interventions and wants to be more involved in the decision making process [19]. Scientific data through research made it possible to predict at a systems level through simulations and models as well as through identification of early on biomarkers for diseases. Similarly prevention through early diagnosis (including family history, risk groups, genetic susceptibility, etc.) and understanding disease progression in an individual with reference samples as well as involving the person in the decision making process brought forward the concept of $\mathrm{P} 4$ (predictive, preventive, personalized and participatory) medicine [20]. However, this was not followed through contrary to the fact that there is now a gradual shift towards personalized interventions in the whole arena of healthcare [21], given the concept of 'one shoe does not fit all'. This transition also evolves from genomics focused personalized medicine to personalized medicine that takes all the relevant factors (including environmental) into account [21], shifting the paradigm from genomics to a systems approach in medicine and healthcare.

Given the dynamic nature of molecular life sciences as a large system (human) as mentioned above, this article aims to touch upon the challenge of encompassing environmental factors affecting biological molecular systems in scientific data and address the issue in personalized medicine of the bottlenecks of real-time integration of systems biology innovations in healthcare systems.

\section{The Pilot}

Computational power in the form of ICT (information and communication technologies) was driven by large scale physics and commercial applications where medicine played a small role [22]. This is now changing as more ICT tools are being used and developed towards medicine. A revolution in ICT can potentially open the door for functional dynamics studies including systems biology applicability in healthcare like it did for other sectors.

The challenge remains largely for ICT to develop tools in the form of hardware and software to compute these already existing large data sets and ongoing generation of data taking into account the dynamic nature of the interactome [17], as well as all factors including socioeconomic, environmental and molecular determinants of health to implement the P4 medicine [20]. The EU pilot flagship project ITFoM (ICT Future of Medicine) is one of the six pilot projects in the 'European Future and Emerging Technologies flagships' and does take this into consideration. ITFoM aims to develop the virtual counterpart of an individual pulling the healthcare towards not just personalized medicine but individualized medicine, given the fact as described before that 'one shoe does not fit all' [21]. The idea is to first simulate and test drugs/medicines on the virtual patient (ICT-based replicas of molecular organization of individual humans) and/or gets advice from this developed ITFoM system and then executing the advice on the actual patient/individual. This can be accessed by both the individual and doctor for solutions. ITFoM and related projects of the "virtual patient" will develop an entirely new ICT that will enable real-time dynamic models of biochemical pathways, cells, tissues, diseases and ultimately, the entire human [22]. The strategy will be based on a common denominator replica [22]. Not only molecular data but all types of medical dataflow will be integrated in the system as well as environmental and lifestyle factors and will be a self-learning, followup system with reference datasets.

\section{Implementation}

However, like genome-based technologies and system biology, studies of functions of molecules or networks in dynamic environments although partly successful, do not see the implementations or direct use of these generated computational data applications in healthcare systems. Even if ICT does give a push in the right direction and we are able to develop the virtual patient there still seems to be a roadblock. This is given the assumption that the logical roadblock itself viz., the complexity of data and functionality in a dynamic environment is well understood or statistically significant and the environmental factors are acceptably taken into account. Furthermore, the ethical, legal, economic as well as privacy issues are considered. Although ICT and 
related projects may be the forerunners of taking into account to some extent ethical, legal, economic as well as privacy and governance issues there are implications beyond the obvious, which are generally an oversight and a common mistake of the academic-industrial complex [23].

ICT tools have been developed for medicine but its practical usage for most parts is far away. It can be also said that ICT tools developed for medicine, were limited to just molecular interactions and not taking into account general medical, environmental and lifestyle factors which greatly influence the outcome. ITFoM addresses these issues among others, however, from patterns $[24,25]$, in technological integration in healthcare systems [26], it seems evident technological diffusion is a long process [24]. ITFoM and related projects will set the stage for an ICT revolution in medicine. Learning from history, ICT innovations like health technologies, in general lack to capitalize on the issues of healthcare integration or translation, in a timely as well as effective and efficient manner [24,27,28].

Public health and healthcare systems are a different system compared to tools (including technologies) developed for them. Coming back to the oversight or common mistake and the reason for the above mentioned pattern of delayed implementation, is that when developing technologies or knowledge discovery for transferring to healthcare systems, one generally neglects the health policy aspects of the healthcare system [29]. The commonly used activity for such translation [30,31], is technology transfer (TT), which is seen as the activity of the migration of academic discoveries to useful application in the development of marketable products or processes [32]. When valorizing new technologies focus is generally to the market itself and stays there. The push for healthcare implementation stagnates as a result and can be very long say 20 years [24]. Scientific merit or just evidence is not sufficient for real time uptake by healthcare. For example, the technology's clinical utility, which is a measure of the health care value provided by the technology [33], is of relevance, as well as the market authorization, reimbursement and insurance. Unheard terms like Health Needs Assessment (HNA), Health Technology Assessment (HTA) and Health Impact Assessment (HIA), collectively known as the Public Health Assessment Tools (PHAT) [29,34,35], play an important role in healthcare implementation and are used by health professionals, doctors, decision makers or policy makers and are detrimental to insurance and reimbursement policies regarding a technology.

HNA is a systematic method of reviewing the health issues facing a population, leading to agreed priorities and resource allocation that will improve health and reduce inequalities [34]. HTA is a multidisciplinary process that summarizes information about the medical, social, economic and ethical issues related to the use of a health technology in a systematic, transparent, unbiased, robust manner. Its aim is to inform the formulation of safe, effective, health policies that are patient focused and seek to achieve best value [36]. This corresponds to interventions and involvement in appraisals making HTA an influential tool to inform decision-making [29]. HIA is a combination of procedures, methods and tools by which a policy, program, or project may be judged as to its potential effects on the health of a population, and the distribution of those effects within the population [37]. In summary, HNA identifies health priorities for a given population, HTA evaluates the performance of health care technologies and HIA assesses the effects of policies, programs or projects on the population's health [34]. The above mentioned terms are generally neglected during technology transfer resulting in healthcare implementation delay [29].
Recently to resolve the bottleneck or roadblock a new framework called the LAL (Learning-Adapting-Leveling) model has been developed addressing healthcare implementation through and to the end of the technology transfer activity [29]. The LAL model's core (Figure 1) lies at the pseudo-parallel initiation of TT and PHAT through bilateral communication, feedback, public-private partnerships, and consultation between the two enterprises to adapt, stop or further the developing relevant technology based on its value to healthcare. Thus saving time and resources and accomplishes in real time by minimizing the delay of non or mis-communication between the different fields i.e. technology transfer and PHAT. Also, early on involvement of all stakeholders including the academic-industrial complex [23], health professionals, doctors, insurance companies, policy makers, patient groups, investors, etc. is within the framework of the model. In addition the 10 essential tasks of the Public Health wheel [38], which will ensure genome-based information and technologies, can be integrated into public health are covered. The LAL model brings two different fields of work for the first time together, i.e. technology transfer (TT) and the public health assessment tools (PHAT) and early on involvement of all stakeholders, public health integration and the value of information generated [29]. Ethical legal, social, economic aspects and clinical as well as personal utility [39-41], among others are covered in the model [29]. The model operates as a feedback mechanism to adapt and integrate new information and compensate through its collaborative communication network. It works as an overarching framework among frameworks within and between difference enterprises.

ITFoM also takes into consideration healthcare integration issues by following the LAL model in its implementation plan. Although the

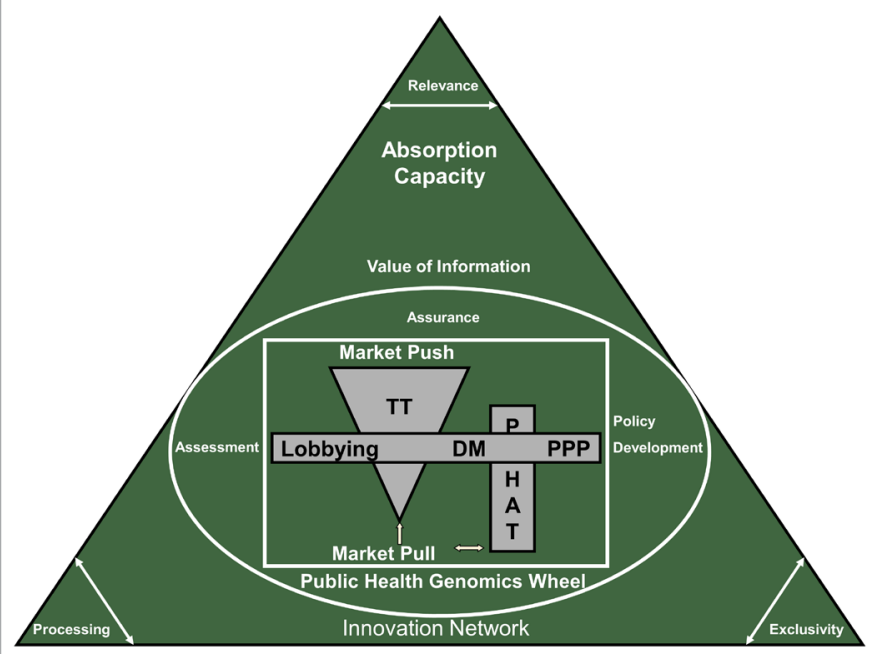

Figure 1: The Learning-Adapting-Leveling ( $L A L)$ model-The $L A L$ model is a framework, which addresses the issue of real-time integration in healthcare systems. The added value of the model is that for the first time it brings together technology transfer and the public health assessment tools in parallel working together by crosstalk (via public-private partnerships or PPPs), giving rise to the innovation network, and in the process adapting the technology to public health and society needs. By the time the technology roles out of the TT pipeline, it is well suited to the conformity of PHAT, and to be taken up by decision makers (DM). This model takes into consideration, the 10 essential tasks of the public health wheel in the form of its 3 domains, namely policy development, assessment and assurance. The model also takes into account the Value of Information from the perspective of the developing technology's relevance to the end consumer, its processing ability or understandability to the user, and exclusivity like intellectual property rights, which may restrict its wide usage. This (innovation) network through PPPs has an optimal working function called the absorption capacity [29]. 
LAL model sets the stage for real-time uptake of relevant technologies and information data for healthcare implementation and the primordial soup for new policies in that direction, the development of policies favoring relevant uptake is an important aspect. Based on these polices which in part are contributed through recommendations of HTA reports, actual implementation and acceptance of health technologies or health information in healthcare settings are executed and for the technologies, reimbursement and market authorization is released. Policy tools in the form of guidelines have been developed by the Public Health Genomics European Network [42], (www.phgen.eu ) complimenting ITFoM and the LAL model.

The Public Health Genomics European Network (PHGEN II) is a European Commission DG SANCO (Directorate General for Health and Consumer Affairs) supported project and constitutes partners from all European Member States and observers. The aim of PHGEN II is to develop European best practice guidelines for quality assurance, provision and use of genome-based information and technologies by different stakeholders at an EU level [42]. The scope of PHGEN II is to assist EU Member States among others to develop relevant policies for-omics integration in healthcare. These guidelines [42] are a meta level guidance tool to further develop guidelines and are based around the 10 essential task of the Public Health Wheel [38], as can be seen from figure 2, which strives to integrate genome-based information and technologies into public health. This brings in the field of Public Health Genomics, which is the responsible and effective translation of genome-based knowledge and technologies into public policy and health services for the benefit of population health [43]. With final dissemination of these guidelines to all Member States and advocacy for policy implementation as well as set up of PHGEN National Task Forces will help towards healthcare implementation of ITFoM and the LAL model.

\section{Discussion and Conclusions}

Although functional dynamics as just a field of study can exist as a research theme within systems biology, however for it to be useful to medicine, personalized medicine, personalized healthcare and health-

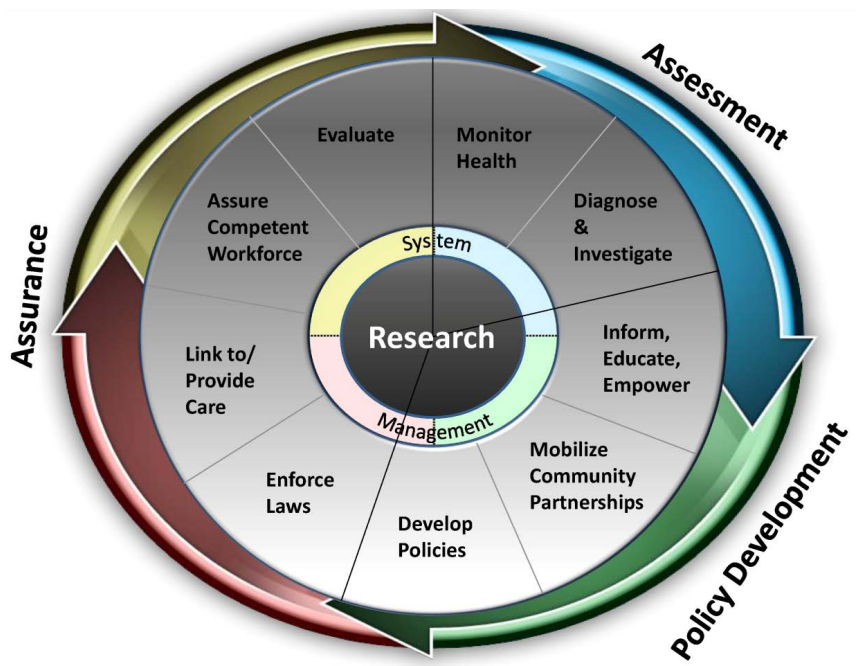

Figure 2: The 10 essential Public Health tasks-The 10 mentioned tasks are given in the pie chart divided over the 3 public health domains of assessment, policy development and assurance, with research being the core in all tasks. The European best practice guidelines developed by PHGEN were based around these 10 essential tasks [38]. care as a whole, knowledge generated and subsequent relevant technologies developed need to be readily absorbed by healthcare systems in real-time. In order for that to happen the field while embarking on such an endeavor should not neglect health policy aspects and all stakeholders involved from an early on stage. The platform is set through ITFoM, the LAL model and PHGEN II for scientific, implementation and policy-wise respectively for healthcare uptake. However, these levels or steps can be achieved only once stability has been achieved with regard to the challenges functional dynamics face with biological complexity as previously stated. Moreover, environmental interactions add on to this seemingly chaotic, but actually synergistic complexity. ITFoM and related projects aim to address these two supposedly loose ends.

Another aspect of this implementation is the fact that there is a shift from the 'one shoe fits all' concept of medical classification and from stratified medicine to personalized and possibly individualized medicine given the uniqueness and similarities of individuals; and need to be taken into consideration when advocating for new policies. The LAL model and PHGEN II supports this shift in priorities and is already pre-existent within the core concept of ITFoM and related projects of the "virtual patient".

Systems biology coupled through the power of ICT seems to be the future of medical research and beyond given its systemic complexity and likely will benefit research and application of functional dynamics through magnifying its computational power and inclusion of environmental factors as a standard. Studying functions of molecules and networks without the exposome is indeed incomplete. This research will eventually push for personalized/individualized medical as well as health interventions in the future.

\section{Future Prospects}

Functional dynamics has a bright future given the incremental potential of ITFoM through systems biology. This is just not from a scientific point of view but given the framework of the LAL model and the policy in place through PHGEN II, active uptake by healthcare is a possibility. What remains to be seen is how the ITFoM pilot project develops its models further and integrates data both scientific and environmental to its new ICT as well as developed infrastructure in hardware and software. The impact of the translation of this research and development into healthcare systems is hard to accurately predict given the semi-theoretical nature of what is currently implied, but what can be said is once accomplished it will greatly impact and revolutionize healthcare as a whole. It will change for sure the doctorpatient relationship. ITFoM tools can be used by both patients and doctors among others to be decided, however this will change the role each stakeholder plays and will bring it a new set of ethical, legal and socio-economic implications and organizational changes currently immeasurable.

\section{Acknowledgements}

This project is supported by a grant from the European Commission PHGEN II (Duration period: June, 2009-May 2012 EU-Project No. 20081302). This work is also supported by the Canadian Institutes for Health Research (CIHR), CIHR Institute of Genetics, the ClHR Institute of Health Services and Policy Research through a grant (no. ETG92250), to the APOGEE-Net/CanGèneTest Research and Knowledge Network on Genetic Services and Policy. We also like to extend our thanks to the research schools CAPHRI (program Public Health Genomics) and GROW of the Faculty of Health, Medicine and Life Sciences of Maastricht University for supporting this research.

\section{References}

1. Alon U, Surette MG, Barkai N, Leibler S (1999) Robustness in bacteria chemotaxis. Nature 397: 168-171. 
Citation: Lal JA, Sudbrak R, Lehrach H, Brand A (2013) Functional Dynamics: From Biological Complexity to Translation and Impact in Healthcare Systems. J Comput Sci Syst Biol 6: 088-092. doi:10.4172/jcsb.1000104

2. Yi TM, Huang Y, Simon MI, Doyle J (2000) Robust perfect adaptation in bacterial chemotaxis through integral feedback control. Proc Natl Acad Sci U S A 97: 4649-4653.

3. Weng G, Bhalla US, lyengar R (1999) Complexity in biological signaling systems. Science 284: 92-96.

4. Levine K, Tinkelenberg A, Cross F (1995) Progress in cell cycle research Plenum, New York, USA

5. Jordan JD, Landau EM, lyengar R (2000) Signaling networks: the origins of cellular multitasking. Cell 103: 193-200.

6. Little JW, Shepley DP, Wert DW (1999) Robustness of a gene regulatory circuit. EMBO J 18: 4299-4306.

7. Kitano H (2002) Computation systems biology. Nature 420: 206-209.

8. Goh KI, Cusick ME, Valle D, Childs B, Vidal M, et al. (2007) The human disease network. Proc Natl Acad Sci U S A 104: 8685-8690

9. Altschul SF, Gish W, Miller W, Myers EW, Lipman DJ (1990) Basic loca alignment search tool. J Mol Biol 215: 403-410.

10. Schatz MC, Delcher AL, Salzberg SL (2010) Assembly of large genomes using second-generation sequencing. Genome Res 20: 1165-1173.

11. Gusfield D (1997) Algorithms on strings, trees, and sequences: Computer science and computational biology. Cambridge University Press, USA.

12. Myers CL, Chiriac C, Troyanskaya OG (2009) Discovering biological networks from diverse functional genomic data. Methods Mol Biol 563: 157-175.

13. Goldberg DE (1989) Genetic algorithms in search, optimization and machine learning. (1 ${ }^{\text {st }}$ Edn), Addison-Wesley Longman Publishing Co., Inc, USA.

14. Bishop CM (1996) Neural networks for pattern recognition. (1 ${ }^{\text {st }}$ Edn), Oxford University Press, UK.

15. Navlakha S, Bar-Joseph Z (2011) Algorithms in nature: the convergence of systems biology and computational thinking. Mol Syst Biol 7: 546 .

16. Galas DJ, Hood L (2009) Systems biology and emerging technologies will catalyze the transition from reactive medicine to Predictive, Personalized Preventive And Participatory (P4) medicine. Interdisciplinary Bio Central 1: 1-4.

17. Sanchez C, Lachaize C, Janody F, Bellon B, Röder L, et al. (1999) Grasping at molecular interactions and genetic networks in Drosophila melanogaster using FlyNets, an internet database. Nucleic Acids Res 27: 89-94.

18. Rapport SM, Smith MT (2010) Epidemiology. Environment and disease risks Science 330: 460-461.

19. Sørensen K, Brand H (2011) Health literacy: the essential catalyst for the responsible and effective translation of genome-based information for the benefit of population health. Public Health Genomics 14: 195-200

20. Hood L, Friend SH (2011) Predictive, personalized, personalized, preventive participatory (P4) cancer medicine. Nat Rev Clin Oncol 8: 184-187.

21. Cesuroglu T, van Ommen B, Malats N, Sudbrak R, Lehrach H, et al. (2012) Public health perspective: from personalized medicine to personal health. Per Med 9: 115-119.

22. Lehrach H, Subrak R, Boyle P, Pasterk M, Zatloukal K, et al. (2011) ITFoM-The future of medicine. Procedia Comput Sci 7: 26-29.

23. Crow MM, Tucker C (2001) The American research university system as America's de facto technology policy. Sci Public Policy 28: 2-10.

24. Berwick DM (2003) Disseminating innovations in health care. JAMA 289: 1969 1975.

25. Murtagh J, Foerster V (2009) Managing Technology Diffusion-Discussion Paper. Policy Forum 1-13.

26. Booth-Clibborn N, Packer C, Stevens A (2000) Health technology diffusion rates. Statins, coronary stents, and MRI in England. Int J Technol Assess Health Care 16: 781-786.

27. Mcglynn EA, Asch SM, Adams J, Keesey J, Hicks J, et al. (2003) The quality of health care delivered to adults in the United states. N Engl J Med 348: 26352645.
28. Woolf SH (2008) The meaning of translational research and why it matters. JAMA 299: 211-213

29. Lal JA, Schulte in den Bämen T, Morré SA, Brand A (2011) Public health and valorization of genome-based technologies: a new model. J Transl Med 9: 207.

30. (2011) Business Insights: Trends in Biotechnology Technology Transfer to 2014-Deal volume has doubled since 2008. Reports-research.com, Germany.

31. Rasmussen E (2005) The process of new venture creation in a University setting. Accompanying measures \& survival of new firms: between Darwinism and assistance, Montpellier, France 1-28.

32. Mullins B, Crow EJ (1999) Technology transfer: A Roadmap. College and University Auditor, Association of College and University Auditors.

33. Clinical utility of personalised medicine (2011) National Health and Media Research Council. Australian Government, Australia.

34. Rosenkötter N, Vondeling H, Blancquaert I, Mekel OC, Kristensen FB, et al. (2011) The contribution of health technology assessment, health needs assessment, and health impact assessment to the assessment and translation of technologies in the field of public health genomics. Public Health Genomics 14: $43-52$

35. Becla L, Lunshof JE, Gurwitz D, Schulte In den Bäumen T, Westerhoff HV, e al. (2011) Health technology assessment in the era of personalized health care. Int J Technol Assess Health Care 27: 118-126.

36. HTA Definition [http://www.eunethta.eu/about-us/faq\#t287n73].

37. Health Impact Assessment: Main concepts and suggested approach (1999) Gothenburg consensus paper. European Center for Health Policy, WHO Regional Office for Europe, Brussels, Belgium.

38. Beskow LM, Khoury MJ, Baker TG, Thrasher JF (2001) The integration of Genomics into public health research, policy and practice in the United States. Community Genet 4: 2-11.

39. Public Heath Genomics European Network (2011) PHGEN Interim Report of the Guidance for Quality Assurance of Genome-based Information and Technologies.

40. Public Heath Genomics European Network (2012) European Best Practice Guidelines for Quality Assurance, Provision and Use of Genome-based Information and Technologies. (Part II), European Best Practice Guidelines for Provision of Genome-based Information and Technologies.

41. Public Heath Genomics European Network (2012) European Best Practice Guidelines for Quality Assurance, Provision and Use of Genome-based Information and Technologies. (Part III), European Best Practice Guidelines for Provision of Genome-based Information and Technologies.

42. Brand A, Lal JA (2012) European best practice guidelines for quality assurance provision and use of genome-based Information and technologies: the 2012 Declaration of Rome. Drug Metabol Drug Interact 27: 177-182.

43. Burke W, Khoury MJ, Stewart A, Zimmern RL (2006) The path from genomebased research to population health: Development of an international public health genomics network. Genet Med 8: 451-458. 